This item was submitted to Loughborough's Research Repository by the author.

Items in Figshare are protected by copyright, with all rights reserved, unless otherwise indicated.

\title{
Nonperturbing measurements of spatially distributed underwater acoustic fields using a scanning laser Doppler vibrometer
}

PLEASE CITE THE PUBLISHED VERSION

PUBLISHER

(c) Acoustical Society of America

LICENCE

CC BY-NC-ND 4.0

\section{REPOSITORY RECORD}

Harland, Andy R., Jon N. Petzing, and John R. Tyrer. 2019. "Nonperturbing Measurements of Spatially Distributed Underwater Acoustic Fields Using a Scanning Laser Doppler Vibrometer". figshare. https://hdl.handle.net/2134/3640. 
This item was submitted to Loughborough's Institutional Repository by the author and is made available under the following Creative Commons Licence conditions.

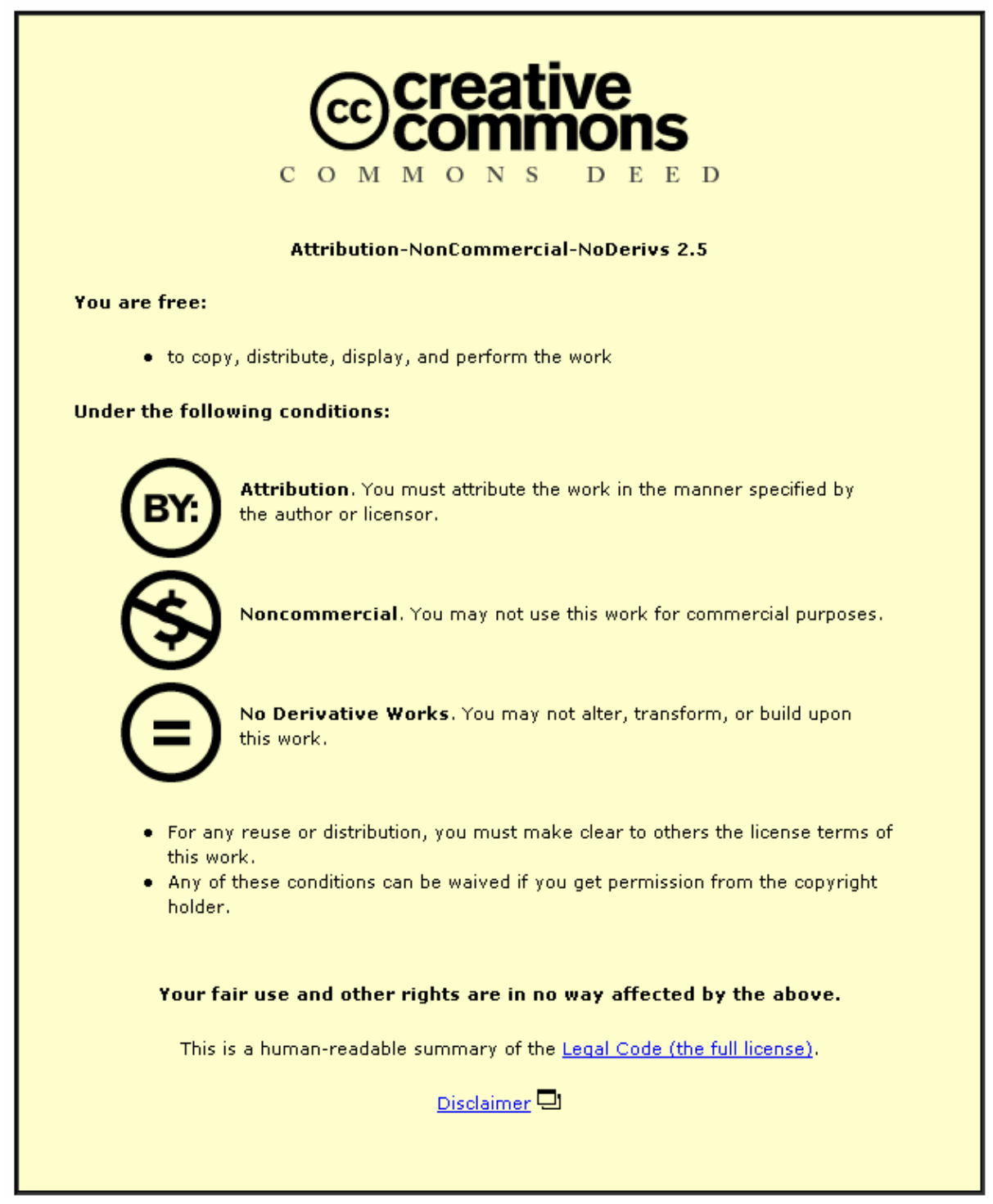

For the full text of this licence, please go to: http://creativecommons.org/licenses/by-nc-nd/2.5/ 


\title{
Nonperturbing measurements of spatially distributed underwater acoustic fields using a scanning laser Doppler vibrometer
}

\author{
Andy R. Harland, ${ }^{\text {a) }}$ Jon N. Petzing, and John R. Tyrer \\ Mechanical and Manufacturing Engineering, Loughborough University, Loughborough, Leicestershire, \\ LE11 3TU, United Kingdom
}

(Received 22 November 2002; revised 22 October 2003; accepted 31 October 2003)

\begin{abstract}
Localized changes in the density of water induced by the presence of an acoustic field cause perturbations in the localized refractive index. This relationship has given rise to a number of nonperturbing optical metrology techniques for recording measurement parameters from underwater acoustic fields. A method that has been recently developed involves the use of a Laser Doppler Vibrometer (LDV) targeted at a fixed, nonvibrating, plate through an underwater acoustic field. Measurements of the rate of change of optical pathlength along a line section enable the identification of the temporal and frequency characteristics of the acoustic wave front. This approach has been extended through the use of a scanning LDV, which facilitates the measurement of a range of spatially distributed parameters. A mathematical model is presented that relates the distribution of pressure amplitude and phase in a planar wave front with the rate of change of optical pathlength measured by the LDV along a specifically orientated laser line section. Measurements of a $1 \mathrm{MHz}$ acoustic tone burst generated by a focused transducer are described and the results presented. Graphical depictions of the acoustic power and phase distribution recorded by the LDV are shown, together with images representing time history during the acoustic wave propagation. (C) 2004 Acoustical Society of America. [DOI: 10.1121/1.1635841]
\end{abstract}

PACS numbers: 43.38.Ne [YHB]

Pages: 187-195

\section{INTRODUCTION}

The propagation of mechanical sound waves in dense media such as water is central to many communication and imaging techniques used in marine and medical applications. The principle of the majority of imaging techniques such as obstetric examination involve relating a measure of the distribution of acoustic energy scattered from boundaries within the volume under interrogation, with a known measure of the acoustic energy provided by the originating source. The quality of the resultant image is largely dependent on the fidelity of these acoustic measurements.

The principal instrument for making underwater acoustic pressure measurements is the hydrophone. A hydrophone typically provides a voltage proportional to the integral of the pressure variation across its active element, although this is generally assumed to be sufficiently small to approximate to a point. Since a hydrophone is required to be submerged in the acoustic field in order to record a measurement, it follows that the field will be perturbed by its presence. Certain design measures, such as ensuring the size of the hydrophone is significantly smaller than the wavelength of the sound being measured and surrounding the hydrophone element with a material of a close impedance matching with water, are used to minimize the perturbation, but it can never be entirely eliminated.

\footnotetext{
${ }^{a)}$ Contact details: A. R. Harland, Mechanical and Manufacturing Engineering, Loughborough University, Loughborough, Leicestershire, LE11 3TU, United Kingdom. Telephone: 01509 227656; fax: 01509 227648; electronic mail: A.R.Harland@lboro.ac.uk
}

In order to characterize the spatial distribution of an acoustic field using a hydrophone, multiple measurements at positions in three orthogonal dimensions are required, either by repeating single hydrophone measurements or establishing a three-dimensional array of hydrophone elements. Both techniques have shortcomings that restrict their application in many instances, for example, the time required to position and record multiple measurements using a single hydrophone and the likely perturbation of the field caused by a threedimensional array of hydrophone elements and their mounting structure.

An example of a system that aims to accurately record the spatial distribution of an acoustic pressure field, and is indeed directly traceable to the primary standard, is the UK's National Physical Laboratory (NPL) Ultrasound Beam Calibrator, used in the frequency range $500 \mathrm{kHz}$ to $20 \mathrm{MHz}{ }^{1}$ Here a 21-element membrane hydrophone made from polyvinylidene fluoride is mounted within an acoustic field and pressure measurements are recorded by each element. The position and orientation of the acoustic source is then modified and measurements repeated. The system offers a compromise where field perturbation and the time required to record measurements are both minimized.

The work reported here is aimed at addressing the need for nonperturbing optical techniques capable of recording spatially distributed measurements from underwater acoustic pressure fields. An overview of relevant previously published work follows, which enables the technique described in this work to be placed into context.

A great many researchers have engaged in the prediction, measurement, modeling, and understanding of the inter- 
action between light and sound. Based on the initial work of Raman and Nath ${ }^{2,3}$ and Debye and Sears ${ }^{4}$ on this subject, several techniques for visualizing sound waves have been developed. These include the apparatus developed by Willard for visualizing ultrasonic waves ${ }^{5}$ and the application of the schlieren method traditionally used for identifying aberrations within large astronomic objective lenses. ${ }^{6}$

A comprehensive description of the theory of acoustooptic interaction is presented by Klein and Cook. ${ }^{7}$ The topic of ultrasonically induced optical diffraction has been the subject of many reviews, two of which were presented by Haran $^{8}$ and Monchalin. ${ }^{9}$ A more recent summary of highfrequency acoustic measurements by optical techniques was authored by Cook. ${ }^{10}$

The advent of coherent light (laser) sources has enabled a refinement in optical diffraction measurements such that Blomme and Leroy were able to establish the second- and third-order approximation methods for characterizing the diffracted light. ${ }^{11,12}$ While each of these techniques offered the ability to provide information regarding the spatial distribution of an acoustic field, the techniques were only able to provide qualitative data.

A departure from the conventional diffraction measurements of light intensity came when Reibold and Kwiek began to measure the amplitude as opposed to the intensity of the light, enabling the complex nature of the various diffracted orders to be investigated. This progression, combined with advances in high-powered computer processing equipment, enabled the development of light diffraction tomography. ${ }^{13}$ In this process the magnitude and phase of the acoustic wave were established by filtered back-projection using discrete convolution of the diffracted light at multiple angles through the field. By this method, a single-point measurement of pressure could be derived. Further development of the technique was then documented ${ }^{14-17}$ such that in their review of 1995, Reibold and Kwiek described light diffraction tomography as being a capable tool for mapping ultrasonic fields up to $5 \mathrm{MHz}$ in frequency. ${ }^{18}$ The technique is, however, limited by the resources and time required to complete a scan and the fact that single erroneous points contribute significantly to the overall uncertainty of the measurement result.

Electronic Speckle Pattern Interferometry (ESPI) was first reported by Leendertz and Butters in $1973^{19}$ and has been applied to the measurement of pressure-induced refractive index changes present in underwater acoustic fields. Having extensively studied refractive index changes induced by airborne acoustic fields emanating from vibrating objects such as loudspeakers using ESPI, ${ }^{20,21}$ the Applied Optics Group at the Norwegian University of Science and Technology successfully implemented the observation of underwater acoustic fields using the same technology. ${ }^{22}$ In a more specific study, Rustad demonstrated the suitability of ESPI for studying the acoustic field generated by a $3.25 \mathrm{MHz}$ continuous wave medical ultrasound probe, with a spatial resolution of better than $100 \mu \mathrm{m} .{ }^{23}$ This technique is, however, limited by the spatial resolution and fixed aspect ratio of the TV camera used to acquire the images and the fact that the sensitivity of the ESPI system is a complex function of the spherical spreading of the optical wave front and target shape and position.

In a similar approach to that taken by Reibold $e t$ al. for light diffraction studies, phase stepping and tomographic reconstruction techniques have been applied to measurements of sound fields in air. ${ }^{24,25}$ The application of a pulsed laser source has enabled transient acoustic waves to be recorded and tomographically reconstructed from ESPI measurements. ${ }^{26}$

The work described here details a method of using a commercially available laser scanning vibrometer to record noninvasive spatially and temporally distributed measurements from underwater acoustic fields. The work advances previous research by allowing two-dimensional whole field images to be produced from arbitrarily shaped acoustic fields.

\section{THEORETICAL ANALYSIS}

Laser Doppler vibrometry (LDV) is a well-established optical metrology tool, conventionally used to record surface velocity measurements from vibrating objects. ${ }^{27}$ Also known by the more generic term of laser Doppler velocimetry, LDV has been previously demonstrated to be effective in recording the temporal distribution of an underwater pressure wave as it passes over the single line section of the laser beam directed through the acoustic volume. ${ }^{28-31}$ Since the instrument used in this work is a commercially available vibrometer, this term is used throughout the work, although it is recognized that the principle by which the instrument records the measurement (by measuring the rate of change of refractive index) is not that by which a conventional vibrometer operates.

Consider the ideal case of a collimated beam of radius $r$, with plane phase fronts. In its simplest geometry, the beam from the LDV is normal to the axis of the sound beam. In this arrangement, the acoustic phase, $\Phi$, remains constant with distance along the line and the voltage output from the $\mathrm{LDV}, V$, which is proportional to the rate of change of optical path length, is described by Eq. (1), where $K$ is the sensitivity scalar of the LDV electronics, $(\partial n / \partial P)_{s}$, is the adia-

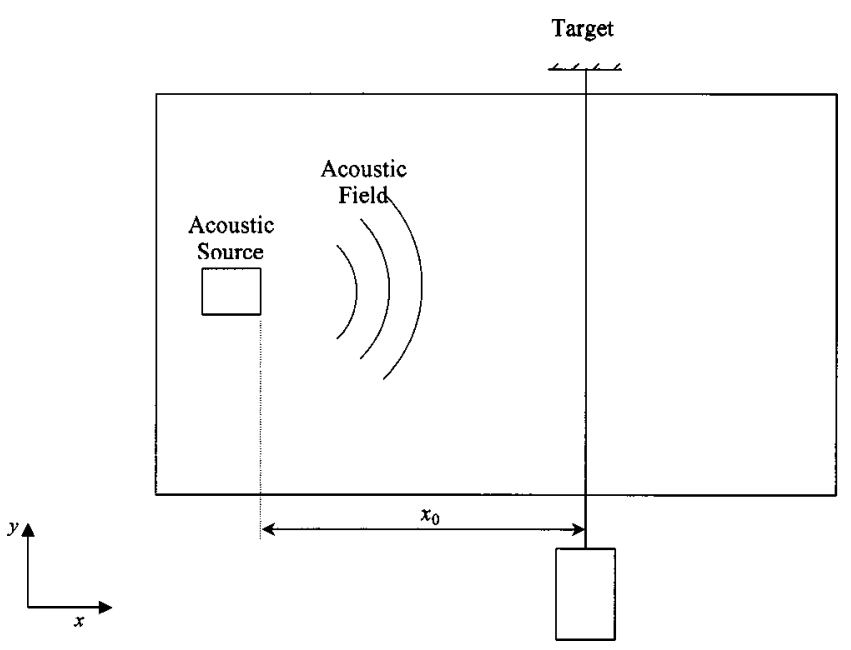

FIG. 1. Experimental arrangement depicting the propagation distance between the acoustic source and the LDV laser beam. 


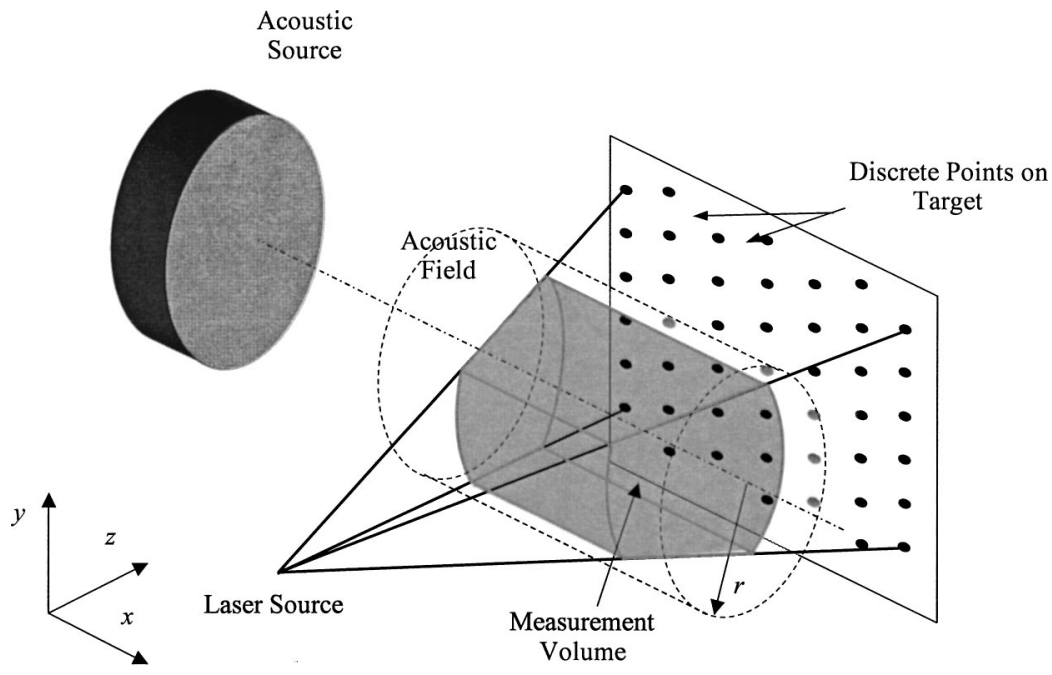

FIG. 2. The interactive range of scanning LDV with the acoustic field, defined by the laser beam at its angular extremities.

batic piezo-optic coefficient, $A$ is the acoustic pressure amplitude, and $f$ is the acoustic frequency. The optical pathlength, $l$, represents the integral of the refractive index, $n$, with distance, where the limits of integration are of the path of the laser beam that is affected by the sound field:

$$
V(t)=K \frac{d l(t)}{d t}=8 \pi r K\left(\frac{\partial n}{\partial P}\right)_{s} A \cos (2 \pi f t-\Phi) .
$$

When the LDV measurement is triggered in time by the input voltage to the transducer, the perpendicular distance from the laser beam position to the front face of the acoustic source, $x_{0}$, determines the time delay prior to the arrival of the acoustic tone burst. This is depicted in Fig. 1.

It follows, therefore, that information regarding the spatial distribution of the acoustic field can be obtained by comparing the rate of change of the optical pathlength measured at a range of laser beam positions with respect to the acoustic source. An extension to this analysis of a single line section through an acoustic field is to consider multiple line sections simulating the position of a laser beam within a twodimensional range.

A scanning LDV system allows the beam to be sequentially directed within a range specified by a number of discrete positions established on a fixed, stationary target be- yond and outside the acoustic volume, as depicted in Fig. 2. Since each measurement is triggered by the electrical input signal to the source, a referenced measure of spatial and temporal pressure distribution can be established. The output from each measurement recorded in this way is likely to vary in two ways. First in phase, predominantly due to the timeof-flight delay prior to the arrival of the acoustic tone-burst and second in magnitude, determined by the position and incidence angle of the laser beam.

Since the angles of incidence cannot be perpendicular to the acoustic axis for more than one measurement within the same scan, an additional mathematical explanation is needed to accommodate for angular discrepancy. Given that the laser beam is directed by two scanning mirrors, each manipulating the horizontal and vertical position of the beam, respectively, it follows that the position of the beam should be defined by angles in two planes; the $x-z$ plane, represented by $\phi$, and the $y-z$ plane represented by $\theta$. A typical arrangement is shown in Fig. 3. The $z$ axis is defined as the line passing from the laser source through the acoustic axis with normal incidence. The $y$ axis remains orthogonal to both the acoustic axis and the $z$ axis.

When the laser beam is incident with arbitrary angles (polar angle $\phi$ and elevation angle $\theta$, as shown in Fig. 3) on

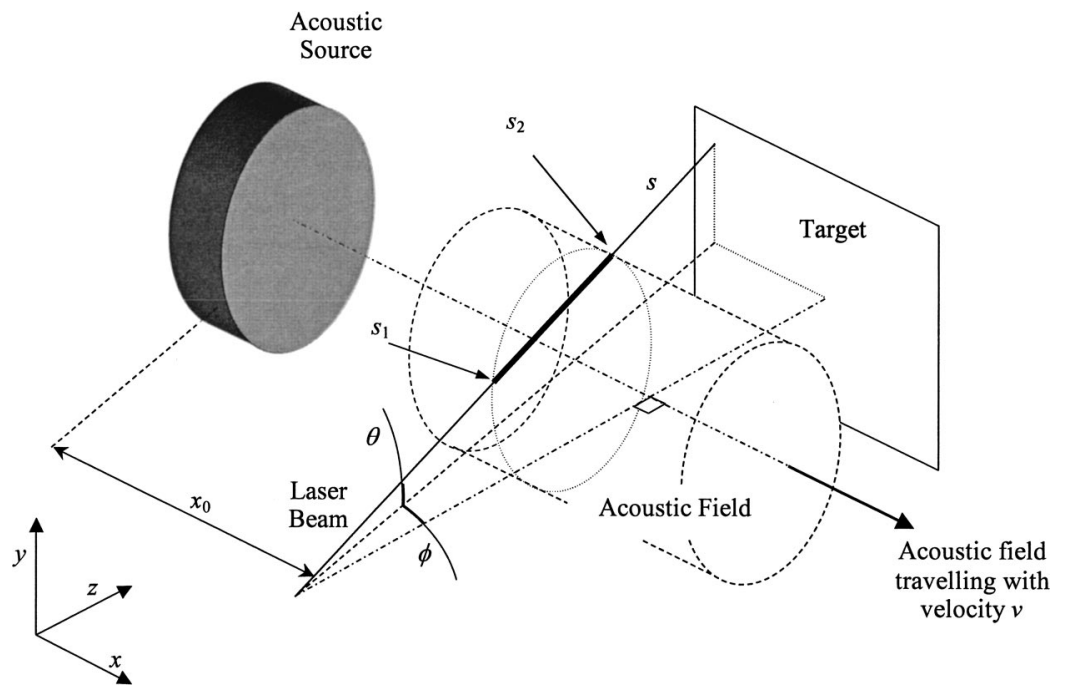

FIG. 3. LDV laser beam passing through an acoustic field with incidence described by the angles $\phi$ and $\theta$. 
the collimated acoustic beam, the optical pathlength, $l$, is written as

$$
l=l_{0}+A_{0}\left(\frac{\partial n}{\partial p}\right) \int_{s_{1}}^{s_{2}} \sin \left[\omega t-k\left(x_{0}+|s| \cos \theta \sin \phi\right)\right] d s,
$$

where $k=\omega / c$ is the acoustic wave number, and $l_{0}$ is the ambient optical pathlength.

The integration leads to

$$
l(t)=l_{0}+A_{0}\left(\frac{\partial n}{\partial p}\right) \frac{1}{\alpha}\left[\cos \left(\alpha\left|s_{2}\right|-\Psi\right)-\cos \left(\alpha\left|s_{1}\right|-\Psi\right)\right],
$$

where $\alpha=k \cos \theta \sin \phi$ is the wave number projected onto the normal axis, and where $\Psi=\omega t-k x_{0}$ is the phase term when the beam is normal to the axis of the sound field. The distances $\left|s_{1}\right|$ and $\left|s_{2}\right|$ are indicated in Fig. 3. They represent the position at the beginning and the end of the acousto-optic interaction region, respectively, and are defined in Cartesian vector form in Eq. (4), where $d_{a}$ is the distance of the acoustic source from the laser source:

$$
\begin{aligned}
\overline{s_{1}} & =\left[\begin{array}{c}
s_{1 x} \\
s_{1 y} \\
s_{1 z}
\end{array}\right] \\
& =\left[\begin{array}{c}
\tan \phi \cos \theta\left(d_{a} \cos \theta-\sqrt{r^{2}-\left(d_{a} \sin \theta\right)^{2}}\right) \\
\sin \theta\left(d_{a} \cos \theta-\sqrt{r^{2}-\left(d_{a} \sin \theta\right)^{2}}\right) \\
\cos \theta\left(d_{a} \cos \theta-\sqrt{r^{2}-\left(d_{a} \sin \theta\right)^{2}}\right)
\end{array}\right],
\end{aligned}
$$

$$
\begin{aligned}
\overline{s_{2}} & =\left[\begin{array}{c}
s_{2 x} \\
s_{2 y} \\
s_{2 z}
\end{array}\right] \\
& =\left[\begin{array}{c}
\tan \phi \cos \theta\left(d_{a} \cos \theta+\sqrt{r^{2}-\left(d_{a} \sin \theta\right)^{2}}\right) \\
\sin \theta\left(d_{a} \cos \theta+\sqrt{r^{2}-\left(d_{a} \sin \theta\right)^{2}}\right) \\
\cos \theta\left(d_{a} \cos \theta+\sqrt{r^{2}-\left(d_{a} \sin \theta\right)^{2}}\right)
\end{array}\right],
\end{aligned}
$$

such that the respective distances from the laser source to the points $s_{1}$ and $s_{2}$ are given by

$$
\begin{aligned}
& \left|s_{1}\right|=\sqrt{-\frac{\left(-d_{a} \cos \theta+\sqrt{r^{2}-d_{a}^{2}+d_{a}^{2} \cos ^{2} \theta}\right)^{2}\left(-\cos ^{2} \theta+\cos ^{2} \theta \cos ^{2} \phi-\cos ^{2} \phi\right)}{\cos ^{2} \phi}}, \\
& \left|s_{2}\right|=\sqrt{-\frac{\left(d_{a} \cos \theta+\sqrt{r^{2}-d_{a}^{2}+d_{a}^{2} \cos ^{2} \theta}\right)^{2}\left(-\cos ^{2} \theta+\cos ^{2} \theta \cos ^{2} \phi-\cos ^{2} \phi\right)}{\cos ^{2} \phi}} .
\end{aligned}
$$

Differentiating the total optical pathlength, $2 l$, with respect to time to calculate the rate of change of optical pathlength as measured by the LDV, gives the following:

$$
\begin{aligned}
\frac{d l(t)}{d t}= & 2 A_{0}\left(\frac{\partial n}{\partial p}\right) \frac{\omega}{\alpha}\left[\sin \left(\omega t-\left(k x_{0}-\left|s_{1}\right| \alpha\right)\right)\right. \\
& \left.-\sin \left(\omega t-\left(k x_{0}-\left|s_{2}\right| \alpha\right)\right)\right] .
\end{aligned}
$$

From Eq. (6) it can be seen that the sensitivity of a LDV laser beam is dependent on its angular position with relation to the acoustic axes, $\theta$ and $\phi$, and the frequency, $f$, of the acoustic wave. Due to the presence and relative positions of these variables within Eq. (6), it follows that at certain angular positions, the pressure distribution will be such that the integral of refractive index with distance along the laser beam through the interactive region is equal to zero. This will result in the optical pathlength of the laser beam remaining constant with time. Hence, at certain pairs of angles $\theta$ and $\phi$, the LDV, which provides a measure of the rate of change of optical pathlength, will be totally insensitive to the acoustic wave.

One measure that can be taken to minimize the influence of the angular position of the beam is to ensure that the stand-off distance between the scanning head and the acoustic field is sufficiently greater than the range of the scan to enable each beam position to be approximately parallel.
The theoretical rate of change of optical pathlength described in Eq. (6) is valid only for LDV interrogations of continuous wave acoustic signals. These are not generally used in laboratory-based underwater acoustic studies due to the need to eliminate interference effects between the signal and other portions of the signal reflected from the tank walls, surfaces, or other impeding obstacles. For this reason, toneburst acoustic signals are used. These consist of typically between 5 and 50 cycles of a single frequency separated by a delay sufficient for the reflected components of the wave to die away.

While the mathematical description of the rate of change of the optical pathlength given in Eq. (6) holds true for the scenario shown in part (b) of Fig. 4, where the interactive region of the laser beam extends from the near to the far side of the acoustic field, it does not describe the measured effect depicted in parts (a) and (c). In both these scenarios, the interactive region is reduced from that of the mathematical description.

To account for the period before and after interaction with the steady-state region of the acoustic wave, one of two approaches can be taken. The first is to consider the tone burst to be a continuous wave but relate the limits of the integral, $s_{1}$ and $s_{2}$, to the position of the tone burst in time and space, such that the distance over which the integral is taken, $\left(s_{2}-s_{1}\right)$, is equal to zero before and after the acoustic 


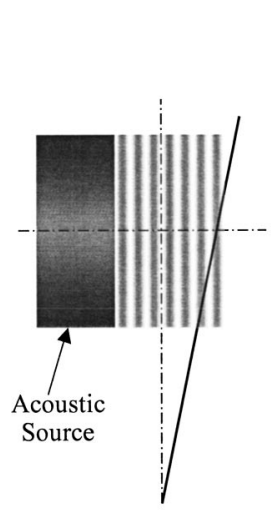

(a)

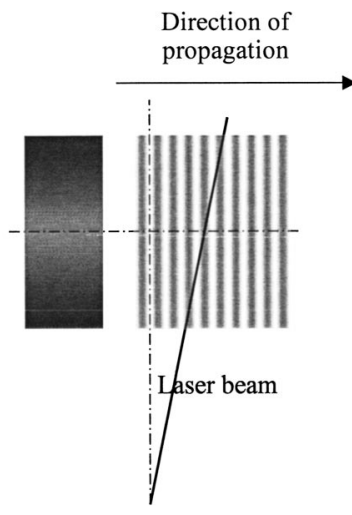

(b)

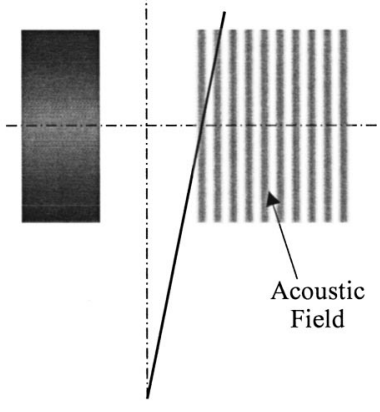

(c)
FIG. 4. LDV laser beam traversing a propagating acoustic tone burst. tone burst has passed. The second approach is to modify the description of pressure from a continuous wave into a tone burst by multiplying the signal by a rectangular window of an appropriate duration in the time domain.

In practice, it is extremely unlikely that the wave front from a plane-piston acoustic source will exhibit entirely plane wave characteristics. Instead, the amplitude, phase, shape, and divergence of the acoustic field will mean that the description of pressure variation with time at a point will be significantly more complex than that used for the mathematical description presented so far. In addition, there are many other designs of acoustic transducer in industrial use, each of which enables the generated field to exhibit certain characteristics.

One such design in widespread use in the medical community is the focused transducer. Here, a concave transducer face generates a spherical wave front, which reduces to a minimum cross section at a focal point some distance away from the transducer. The relationship between the amplitude and phase of the pressure variation with spatial position in three dimensions in a focused field would require the mathematical description of the rate of change of an optical pathlength given in Eq. (6) to take a more generic form, as given in Eq. (7):

$$
\frac{d l(t)}{d t}=2 \frac{d}{d t}\left[\left(\frac{\partial n}{\partial p}\right) \int_{s_{1}}^{s_{2}} A(s) \cos (\omega t-\Phi(s)) d s\right],
$$

where $A(s)$ is the amplitude and $\Phi(s)$ is the phase, both as an unknown function of distance, $s$.

Given the presence of unknown amplitude and phase components within Eq. (7), it follows that without knowledge of the acoustic field, it is impossible to derive pressure amplitude and phase components from experimentally measured data. It is also recognized that in the experimental results that follow, no field can be considered perfectly planar. However, this does not appear to hinder the measurement technique, which provides acceptable visualizations from nonuniform fields.

This mathematical analysis is presented to assist in the interpretation of the results that follow. Since each measurement represents an integral of the refractive index with distance, care should be taken when considering quantities such as acoustic pressure with respect to the experimental results.

\section{EXPERIMENTAL RESULTS}

The experimentation reported here was carried out using a PSV-300 series scanning LDV system manufactured by Polytec GmbH. The system consisted of a scanning head from which the optical measurement laser beam was emitted in a direction controlled by two orthogonal scanning mirrors, associated hardware, and PC-based control software. Integrated within the scanning head was a Charge Couple Device (CCD) TV camera, which facilitated remote setup and provided reference images onto which graphical results could be superimposed.

The experimental arrangement was set up as shown in Fig. 5, with the LDV scanning head positioned approximately $1 \mathrm{~m}$ from the acoustic axis. The laser beam was passed through the front glass wall of the tank, through the measurement volume, through the rear glass wall, and returned along the same path from the stationary target. The target consisted of a rigid plate upon which was mounted a $100 \mathrm{~mm} \times 100 \mathrm{~mm}$ panel of retroreflective material, consisting of a layer of microscopically smooth glass beads adhered to a mirrored backing. A grid of specified increments in $x$ and $y$ was then established on the target, the nodes of which defined the measurement positions for the laser beam. The angular deflections of each of the scanning mirrors were automatically recorded at each of the nodal measurement position.

At each target position, a measurement of the rate of

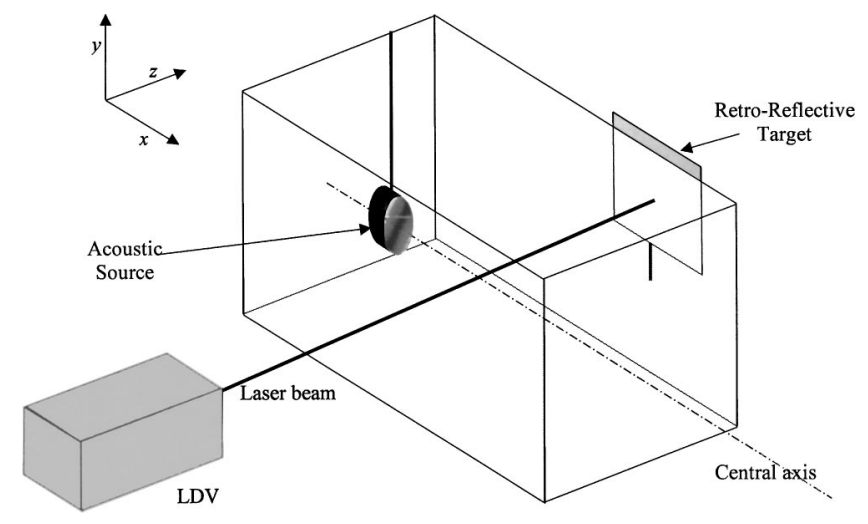

FIG. 5. Experimental arrangement used to record measurements from underwater acoustic fields. 


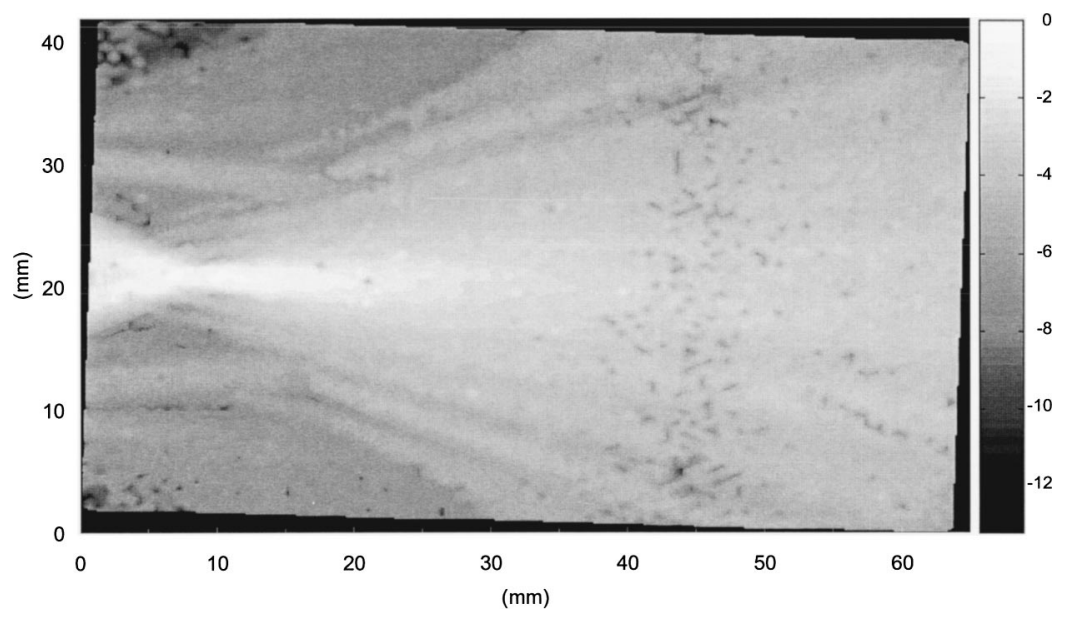

FIG. 6. "Power" distribution of the rate of change of the optical pathlength $\left(\log \mathrm{m}^{2} / \mathrm{s}^{2}\right)$.

change of the optical pathlength was recorded, triggered in time from the electrical input signal to the acoustic source. The distance of the measurement position from the source, the acoustic frequency, and the number of acoustic cycles determined the measurement duration. The signal was sampled at $40 \mathrm{MHz}$ and a Fast Fourier Transform (FFT) with a maximum resolution of 6400 lines was recorded in software.

Since the conventional application of a LDV is to record velocity measurements from a target, the software provided with the scanning system is programed to interpret the measured data as target velocity measurements, which was not the case in the experiments described here. These data are then used to provide a range of graphical representations of the motion and frequency response of the target. For this reason, the original rate of change of pathlength data recorded by the vibrometer were extracted and processed using Matlab to enable measurements of the acoustic field within the water to be derived.

The color or gray-scaled images are widely used to represent the spatial distribution of any distributed parameter, where a specific value representative of the quantity under scrutiny is calculated for each measurement position and presented as a shaded pixel. When each pixel is positioned relative to the neighboring measurements, the array of individual elements combines to form the image. A graduated scale is then calibrated from the lowest to the highest pixel value contained within the image.

Data recorded from the LDV were used to generate images representative of a number of measured quantities. The previously recorded angular positions were used to position each measurement point within the final image. A linear interpolation was then undertaken between adjacent measurements to increase the number of pixels in each axis by a factor of 5, thus improving the visual quality of the images.

In order to provide an example of the range of information that can be extracted from each LDV scan, an experimental scan was completed of the field generated by a Panametrics V3438 transducer emitting a $1 \mathrm{MHz}$ sinusoidal tone burst consisting of five cycles.

A scanning grid was defined containing a total of 7543 discrete laser target points was established. The grid consisted of 71 odd columns each of 53 points and 70 even columns each of 54 points, offset vertically from the odd columns by half the point separation in an "isometric" arrangement, similar to that depicted in Fig. 2. An average of 15 successively triggered time-swept signals of $50 \mu$ s duration were recorded at a sampling frequency of $5.12 \mathrm{MHz}$, and 400 FFT lines stored in a Universal File format.

A measure of the magnitude or power at a certain frequency within a signal measured using a LDV in this way was established from the respective FFT component of the magnitude or power spectrum at the frequency concerned. Each complex FFT recorded by the LDV was converted into a power spectrum and the component at the fundamental acoustic frequency was taken to represent the "power" of the signal. The "magnitude" was calculated as the square root of the calculated power value. Both magnitude and power are quantities derived from the rate of change of the optical pathlength or velocity and take the units of $\mathrm{m} / \mathrm{s}$. Given only the data recorded by the LDV, it is not possible to derive parameters such as acoustic pressure, intensity, or particle displacement, due to the integration effect previously described. It is anticipated that further research and investigation will provide methods for overcoming this shortcoming. Therefore, to avoid a misunderstanding due to idiosyncratic units, it is convenient to normalize the data of these images, creating dimensionless values between 0 and 1 . Images were presented on a logarithmic scale, since this was found to depict their quantity with improved visual clarity.

The principal reason for the use of tone-burst signals in laboratory-based underwater acoustics is to enable measurements to be made without interference from acoustic components reflected from the walls or floor of the water tank or upper air-water interface. Therefore, for each measurement, the "power" and "magnitude" values were extracted from a $4 \mu$ s portion of the signal coinciding with the center of the acoustic tone burst to minimize the influence of spurious acoustic components. The resultant "power" image is given in Fig. 6.

In this particular example, the reflecting target contained a surface defect (scratch) running from the top to the bottom of the scan approximately one-quarter of the image width from the right hand side. This is the cause of the noisy band observed in this region.

In addition to the "power" or "magnitude" within the 


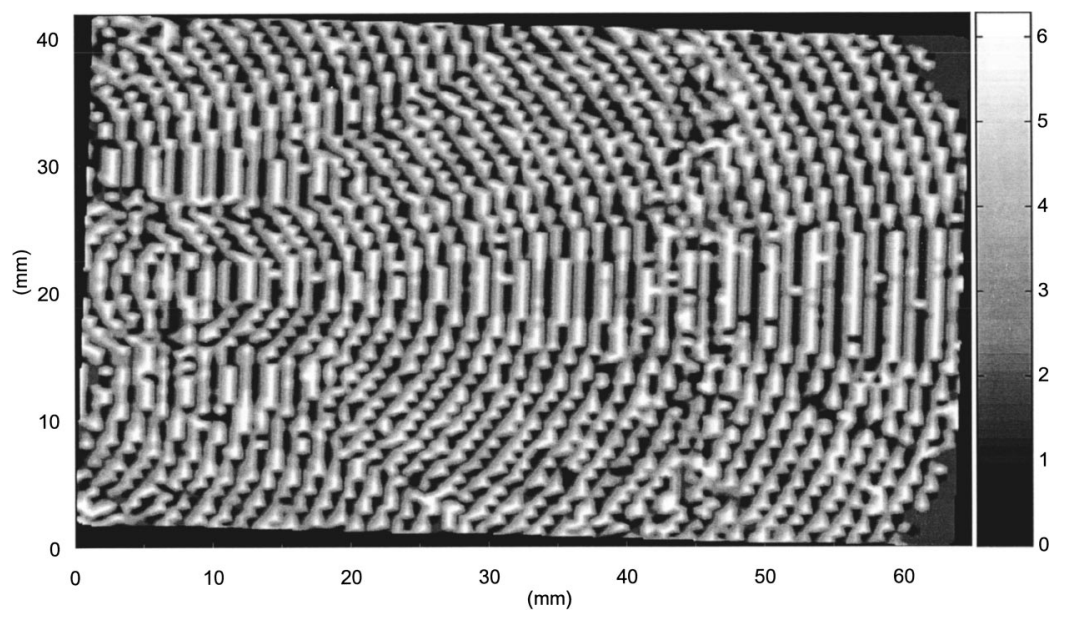

FIG. 7. "Phase" distribution of the rate of change of the optical pathlength (rads).

field, it is important to consider the phase of the amplitude in order to gain a complete understanding of the nature of the acoustic field distribution. Phase data can also be extracted from the measurements; either in terms of the absolute phase-calculated from the arctangent of the quotient of the imaginary component divided by the real component of the FFT - or as a relative phase by converting the position of the tone-burst signal in time with respect to an adjacent point into phase using the period of the signal. The second of these approaches was found to yield the highest quality "phase" distribution images, as shown in Fig. 7.

The most significant observation from the "phase" distribution image is the effect of the spatial sampling resolution on the continuity of the phase distribution. As an absolute minimum, Nyquist's sampling theory states that at least two sampling points per wavelength are required, although it is desirable for this value to be nearer 10 . Given that the
LDV interrogation grid contained 141 discrete columns, the 42 complete cycles observed in the measurement suggest that the spatial sampling rate is toward the lower end of the acceptable range. This is evident in the number of apparent discontinuities in certain regions of the image.

From the derived "magnitude" and "phase" quantities, an image can be created depicting a continuous wave acoustic wave passing through the field at a nominal time by multiplying the "magnitude" by the sine of the phase. Images of this type are able to illustrate the relationship between the magnitude and phase throughout the scan region. By creating a sequence of these images each with a phase shift from the previous image, a cyclic animation mimicking the motion of a continuous acoustic wave through the field can be created.

Using the time-resolved rate of change of the optical pathlength data at each point, a three-dimensional array representing the rate of change of the optical pathlength in $x, y$,
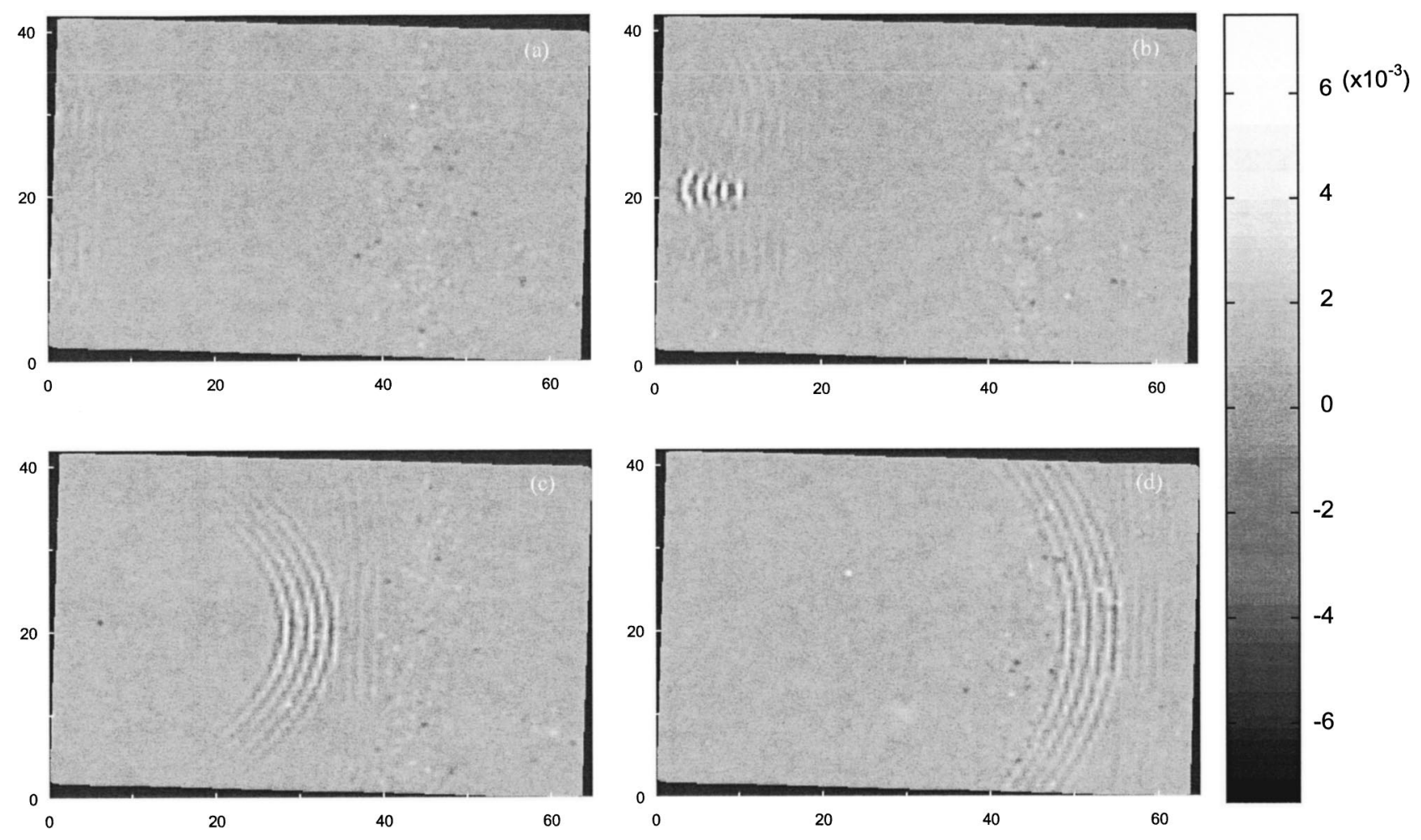

FIG. 8. Time-resolved rate of change of the optical path length $(\mathrm{m} / \mathrm{s})$. 
and time, $t$, can be established. By extracting a plane of data in $x$ and $y$ at a given time, $t_{n}$, a depiction of the position and amplitude of the acoustic tone burst can be obtained at that instant. Four images depicting the acoustic tone burst at different time instants are given in Fig. 8.

The four instants in time at which the acoustic tone burst is depicted in Fig. 8 are 1.5, 50, 130, and $200 \mu$ s after the beginning of the input signal to the acoustic source in (a), (b), (c), and (d), respectively.

Given the ability of the post-processed scanning LDV measurements to enable reconstructions of images at instants in time, it follows that a series of sequential images can be created, which form the individual frames of an animated picture. Consequently, for each LDV scan, an animation can be produced, recreating the passage of an acoustic tone burst through the scanning region. Without interpolation in the time domain, the maximum number of frames constituting the animation is equal to the number of time samples of the recorded signal. An animation created in this way differs from one that can be obtained from the magnitude and phase distribution, in that it represents the true acoustic tone burst, as opposed to a simulated continuous wave.

Previous work has characterized the performance of the Polytec scanning LDV in terms of its minimum resolvable signal amplitude, calculated to be $18.9 \mathrm{mPa} / \sqrt{ } \mathrm{Hz},{ }^{29}$ and its agreement with the NPL Laser Interferometer, measured to be within $2.5 \%$ at $1 \mathrm{MHz}{ }^{29}$ The same LDV has been used throughout the experiments reported in this work.

\section{CONCLUSIONS}

The work reported here describes a nonperturbing optical method for recording spatially distributed measurements of an underwater acoustic field. The instrument has been demonstrated to record optical pathlength changes with time along a finite line section of very small diameter $(<1 \mathrm{~mm})$. By utilizing the refractive index changes induced by the acoustic field, the presence and spatial and temporal distribution of the acoustic tone burst can be clearly identified and quantitatively measured. It has been previously demonstrated that the minimum resolvable signal amplitude is 18.9 $\mathrm{mPa} / \sqrt{ } \mathrm{Hz}^{29}$

A mathematical model has been developed to enable the passage of a LDV beam through an acoustic field to be analyzed. The model is built on well-established physical theory of acousto-optic interaction and developed to cater for the case of a heterodyne or velocity interferometer such as a LDV. The theory has been advanced to permit interrogation at any arbitrary angle of incidence between the optical and acoustic beams

A series of mathematical manipulation and extraction enable images representing the acoustic "power" and "phase" within the acoustic field to be generated. These images provide excellent spatial detail of features, patterns, and discontinuities within the field. Additionally, images depicting the position and magnitude of an acoustic tone burst at an instant in time can be generated.

It should be noted that while a number of quantitative measures of acoustic parameters can be recorded from a re- peatable, arbitrary shaped field, at this stage it is not possible to derive acoustic pressure or intensity at a specific point.

The application of a scanning LDV system has been demonstrated to offer wide-ranging possibilities for the measurement of the spatial characterization of an acoustic field. The ability of the scanning LDV system to rapidly record data from a series of discrete line sections through the field was a significant factor in the establishment of this method as a valuable tool for use in underwater acoustic measurement. It has been successfully demonstrated that the technique allows spatially resolved measurements to be made, which illustrate field components that might otherwise have not been observed.

\section{ACKNOWLEDGMENTS}

The authors gratefully acknowledge the support of Roy Preston and Stephen Robinson from the National Physical Laboratory, UK, for their assistance with the experimentation reported in this work and to Roger Traynor of Lambda Photometrics for the loan of the Polytec scanning LDV and similar equipment.

${ }^{1}$ R. C. Preston, "The NPL ultrasound beam calibrator," IEEE Trans. Ultrason. Ferroelectr. Freq. Control 35, 122-139 (1988).

${ }^{2}$ C. V. Raman and N. S. N. Nath, "The diffraction of light by high frequency sound waves: Part I," Proc. Indian Acad. Sci. 2, 406-412 (1935).

${ }^{3}$ C. V. Raman and N. S. N. Nath, "The diffraction of light by high frequency sound waves: Part II,’ Proc. Indian Acad. Sci. 2, 413-420 (1935).

${ }^{4}$ P. Debye and F. Sears, "On the scattering of light by supersonic waves," Proc. Natl. Acad. Sci. U.S.A. 18, 409 (1932).

${ }^{5}$ G. W. Willard, “Ultra-sound waves made visible," Bell Lab. Rec. 5, $194-$ 200 (1947).

${ }^{6}$ A. Toepler, "Beobachtungen nach einer neuen optischen Methode," Max Cohen und Sohn, Bonn, 1864

${ }^{7}$ W. R. Klein and B. D. Cook, "Unified approach to ultrasonic light diffraction," IEEE Trans. Sonics Ultrason. 14, 123-134 (1967).

${ }^{8}$ M. E. Haran, "Visualization and measurement of ultrasonic wavefronts," Proc. IEEE 67, 454-466 (1979).

9 J.-P. Monchalin, “Optical detection of ultrasound," IEEE Trans. Ultrason. Ferroelectr. Freq. Control 33, 485-499 (1986).

${ }^{10}$ B. D. Cook, "The measurement of high frequency sound fields by optical techniques," SPIE 2358, 278-280 (1994).

${ }^{11}$ E. Blomme and O. Leroy, "Diffraction of light by ultrasound: Closed analytical expression for the spectra up to order 2,' J. Acoust. Soc. India 11, 1-6 (1983).

${ }^{12}$ E. Blomme and O. Leroy, "Diffraction of light by ultrasound: finite analytical expressions for the spectra up to order 3," Acoustica 57, 168-174 (1985).

${ }^{13}$ R. Reibold and W. Molkenstruck, "Light diffraction tomography applied to the investigation of ultrasonic fields. Part I: Continuous waves," Acustica 56, 180-192 (1984).

${ }^{14}$ R. Reibold and P. Kwiek, "On ultrasound light diffraction," Ultrasonics 31, 307-313 (1993).

${ }^{15}$ R. Reibold and P. Kwiek, "Extension of light diffraction tomography beyond the weak acousto-optic interaction," Acustica 81, 43-52 (1995).

${ }^{16}$ P. Kwiek and R. Reibold, "Determination of an ultrasound field using light diffraction," Acustica-acta acustica 82, 376-378 (1996).

${ }^{17}$ R. Reibold and P. Kwiek, "Uncertainty considerations of ultrasonic field mapping by light diffraction tomography," Ultrasonics 35, 187-193 (1997).

${ }^{18}$ R. Reibold and P. Kwiek, "Ultrasound field mapping by light-diffraction tompgraphy: A review," SPIE 2643, 66-79 (1995).

${ }^{19}$ J. A. Leendertz and J. N. Butters, "An image shearing speckle pattern interferometer for measuring bending moments," J. Phys. E 6, 1107-1110 (1973).

${ }^{20}$ O. J. Lokberg, "Sound in flight: measurement of sound fields by use of TV holography,” Appl. Opt. 33, 2574-2584 (1994).

${ }^{21}$ O. J. Lokberg, "Recording of sound emission and propagation in air using 
TV holography," J. Acoust. Soc. Am. 96, 2244-2250 (1994).

${ }^{22}$ R. Rustad, O. J. Lokberg, H. M. Pedersen, K. Klepsvik, and T. Storen, "TV holography measurements of underwater acoustic fields," J. Acoust. Soc. Am. 102, 1904-1906 (1997)

${ }^{23} \mathrm{R}$. Rustad, "Acoustic field of a medical ultrasound probe operated in continuous wave mode investigated by TV holography," Appl. Opt. 37, $7368-7377$ (1998).

${ }^{24}$ O. J. Lokberg, M. Espeland, and M. Pedersen, “Tomography reconstruction of sound fields using TV holography," Appl. Opt. 34, 1640-1645 (1995).

${ }^{25}$ M. Espeland, O. J. Lokberg, and R. Rustad, "Full field tomographic reconstruction of sound fields using TV holography," J. Acoust. Soc. Am. 98, 280-287 (1995).

${ }^{26} \mathrm{P}$. Gren, S. Schedin, and X. Li, "Tomographic reconstruction of transient acoustic fields recorded by pulsed TV holography," Appl. Opt. 37, 834840 (1998).
${ }^{27}$ D. C. Williams, in Optical Methods in Engineering Metrology (Chapman and Hall, London, 1993), ISBN 0-412-39640-8.

${ }^{28}$ A. R. Harland, J. N. Petzing, and J. R. Tyrer, "Non-invasive measurements of underwater pressure fields using laser Doppler velocimetry," J. Sound Vib. 252, 169-177 (2002).

${ }^{29}$ A. R. Harland, J. N. Petzing, J. R. Tyrer, C. J. Bickley, S. P. Robinson, and R. C. Preston, "Application and assessment of laser Doppler velocimetry for underwater acoustic measurements," J. Sound Vib. (in press).

${ }^{30}$ A. R. Harland, J. N. Petzing, and J. R. Tyrer, "Assessment and Application of LDV to underwater acoustic measurements," Proceedings of Photon '02-Applied Optics and Optoelectronics Conference, Cardiff, UK, September 2002, pp. 47-48.

${ }^{31}$ A. R. Harland, J. N. Petzing, and J. R. Tyrer, "Non-invasive measurements of spatially and temporally distributed underwater acoustic pressure fields using laser Doppler velocimetry," accepted for presentation, Institute of Acoustics Conference, Teddington, January 2003. 